\title{
Plasma Adsorption Membranes Are Able to Efficiently Remove High Mobility Group Box-1 (HMGB-1)
}

\author{
Takayuki Onohara ${ }^{1}$, Yuichiro Sakamoto ${ }^{2}$ and Satoshi Inoue ${ }^{3}$ \\ ${ }^{1}$ The Department of Emergency and Critical Care, Ureshino Medical Center, Saga, Japan \\ ${ }^{2}$ The Department of Emergency and Critical Care Medicine, Saga University Hospital, Saga, Japan \\ ${ }^{3}$ The Division of Trauma Surgery and Surgical Critical Care, Saga University Hospital, Saga, Japan
}

\begin{abstract}
Background: High Mobility Group Box 1 (HMGB-1) is a $30 \mathrm{kDa}$ protein that is a lethal mediator in sepsis and is a recognized therapeutic target. However, no consensus has been reached for acute blood purification therapy as a treatment for sepsis targeting HMGB-1. Previous studies demonstrated that a high anti-HMGB-1 antibody titer and the suppression of HMGB-1 activity improved survival rate in animal sepsis models. The aim of this study was to evaluate whether plasma adsorption therapy is able to decrease the level of HMGB-1, representing a new potential treatment strategy against sepsis.

Methods: Plasma adsorption therapy has been known as a treatment for various autoimmune diseases. Three different adsorbent columns, including TR-350 (IM-TR), PH-350 (IM-PH), and BRS-350 (BRS), were used in this study for comparison.

We made a 1/350 scale of these three columns. Fetal bovine serum (FBS) contains a significant amount of HMGB-1. After priming with saline, FBS was passed through the columns and the adsorption rates of HMGB-1 at 25 minutes, 50 minutes, and 75 minutes were evaluated. The total adsorbed HMGB-1 level at 75 minutes was also calculated.

Results: The highest adsorption rate and total adsorbed amount of HMGB-1 were observed in IM-TR, followed by BRS and IM-PH. IM-TR showed a decline in adsorption rate over time. BRS showed a steady adsorption rate at all time points. IM-TR removed HMGB-1 significantly more than IM-PH and BRS.

Conclusions: Our findings showed that plasma adsorption therapy efficiently adsorbed HMGB-1 and could be safely applied for the treatment of sepsis. (J Nippon Med Sch 2018; 85: 150-156)
\end{abstract}

Key words: HMGB-1, sepsis, plasma adsorption, charge, hydrophobicity

\section{Introduction}

Various mediators are known to play a role in sepsis and septic shock. In severe sepsis, pathogen-associated molecular patterns (PAMPs), such as lipopolysaccharide (LPS), are recognized by pattern recognition receptors such as toll-like receptors (TLRs) and promote cytokine production $^{1,2}$. As a result of this inflammatory cascade, tissue and organ damage can occur. In addition to PAMPs, alarmins like HMGB-1 have been found to bind to pattern recognition receptors causing inflammation ${ }^{3,4}$. That is, even if PAMPs are not present, alarmins can amplify inflammation. PAMPs and alarmins are collectively called damage-associated molecular patterns (DAMPs), and this biological reaction is called the damage chain reaction $^{5}$.

In severe sepsis, it is extremely important to control DAMPs, including HMGB-1. The amount of HMGB-1 is significantly correlated with the disseminated intravascular coagulation (DIC) score and the sepsis-related organ failure assessment (SOFA) score. ${ }^{6}$ HMGB-1 is a $30 \mathrm{kDa}$ indispensable protein, which was discovered as a DNA binding protein and is a mediator of cell death when it circulates through the body ${ }^{7}$. It has also been identified as one of the lethal mediators at the late stage of sepsis

Correspondence to Takayuki Onohara, The Department of Emergency and Critical Care, Ureshino Medical Center, 2436 Shimojuku Ureshino, Saga 843-0393, Japan

E-mail: m05019to@jichi.ac.jp

Journal Website (http://www2.nms.ac.jp/jnms/) 


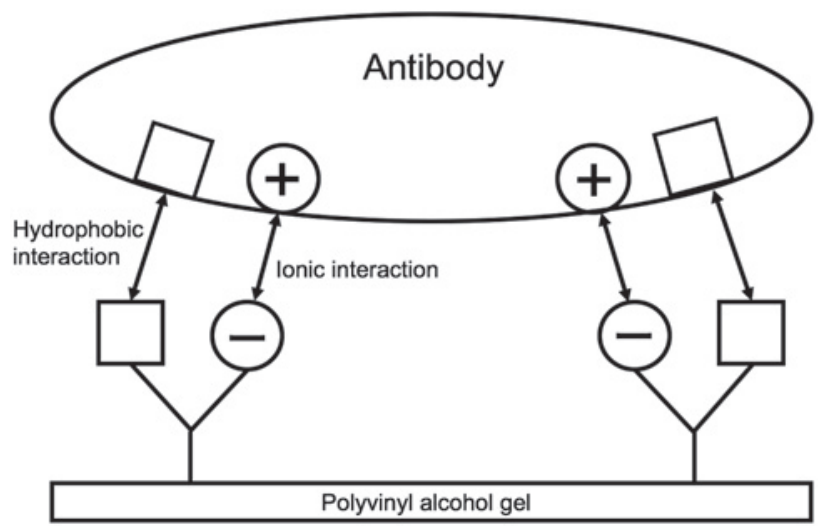

Fig. 1 High Mobility Group Box-1 (HMGB-1) is adsorbed due to both ionic and hydrophobic interactions with adsorbent.

by Wang et al. and HMGB-1 related inflammation has been shown to be enhanced in the presence of interleukin $1 \beta$ and LPS $^{8,9}$. Thus, it is gaining increasing attention from scientists as a potential therapeutic target. The efficiency of sepsis treatment is limited when only single molecules or receptors, such as LPS or the TLR4 receptor, are targeted ${ }^{10,11}$. Thus, blood purification therapy that can remove multiple DAMPs is considered more effective in treating sepsis ${ }^{12}$.

There are treatment guidelines for sepsis and septic shock, such as the surviving sepsis campaign guidelines $(\mathrm{SSCG})^{13}$ and the Japanese guidelines for the management of sepsis ${ }^{14}$. However, acute blood purification therapy, such as polymyxin-B immobilized column direct hemoperfusion (PMX-DHP) and continuous hemodiafiltration (CHDF), has remained controversial as a treatment option for sepsis.

An aberrant activation of HMGB-1 in sepsis should be controlled and adsorption therapy has been reported to be one of the promising mechanisms to reduce these levels $^{15}$. HMGB-1 is a $30 \mathrm{kDa}$ protein, but existing filtration membranes are not capable of efficient removal of substances bigger than $20 \mathrm{kDa}$. Alternatively, plasma adsorption therapies using different columns have been clinically applied for various autoimmune diseases. However, these columns were never tested to reduce the HMGB-1 level in patients with sepsis.

Thus, the purpose of this study was to evaluate whether HMGB-1 can be efficiently adsorbed by columns that have been utilized for autoimmune diseases.

\section{Materials and Methods}

\section{Tools and Devices}

Plasma adsorption therapy is a treatment for various autoimmune diseases such as Myasthenia gravis, Guillain-Barre syndrome and Multiple sclerosis ${ }^{16,17}$. It is also used to treat fulminant hepatitis and postoperative liver failure. In plasma adsorption therapy, blood is drawn and passed through a plasma separator. Then, the separated plasma is brought into contact with the adsorbed column to remove etiologic substances ${ }^{18}$. The plasma with significantly reduced etiologic substances is put back into the body.

Three columns with different adsorption ligands, including TR-350 (IM-TR), PH-350 (IM-PH), and BRS-350 (BRS), were used in this study for comparison. All of the columns were produced by Asahi-kasei medical CO., LTD, Japan. The adsorption ligand of IM-TR was tryptophan, while the adsorption ligand of IM-PH was phenylalanine ${ }^{19,20}$. IM-TR adsorbs anti-acetylcholine receptor antibody, while IM-PH adsorbs immune complex and rheumatoid factor, and anti-DNA antibody, selectively. BRS is fixed with styrene-divinyl benzene as an anion exchange resin. BRS adsorbs bilirubin in fulminant hepatitis and liver failure.

The purpose of plasma adsorption therapy is to capture pathogenic substances by both hydrophobic and ionic interactions ${ }^{20,21}$ (Fig. 1). Both the IM-TR and IM-PH have a hydrophobic group and a carboxyl group with a negative charge. The main material of BRS is polystyrene which contains phenyl groups, making it hydrophobic.

In this study, we made a 1/350 scale of each column, which is $41 \mathrm{~mm}$ in length and $9 \mathrm{~mm}$ in diameter. The major axis of the filter is at $18 \mathrm{~mm}$, with a pore size of $10 \mu \mathrm{m}$. The amount of adsorbent in a $1 / 350$ scale column is $1 \mathrm{~mL}$.

\section{Experimental Procedure}

FBS contains a significant amount of HMGB-1. Experiments were carried out using commercially available bovine serum purchased from SIGMA-ALDRICH, Tokyo, Japan. We first primed the columns by passing $2.86 \mathrm{~mL}$ of saline through them. The plasma flow rate was 0.057 $\mathrm{mL} / \mathrm{min}(3.42 \mathrm{~mL} / \mathrm{H})$ and the amount of plasma processed was $4.28 \mathrm{~mL}$.

HMGB-1 values in FBS before injection, at 25 minutes, 50 minutes, and at 75 minutes following the injection were evaluated and the HMGB-1 adsorption rates at each time point were calculated. All experiments were triplicated using three different columns and average HMGB1 removal ratios were calculated. Additionally, the total adsorbed amount of HMGB-1 in each column was determined, and the adsorption efficiencies of each column were compared. In this experiment, FBS was passed 


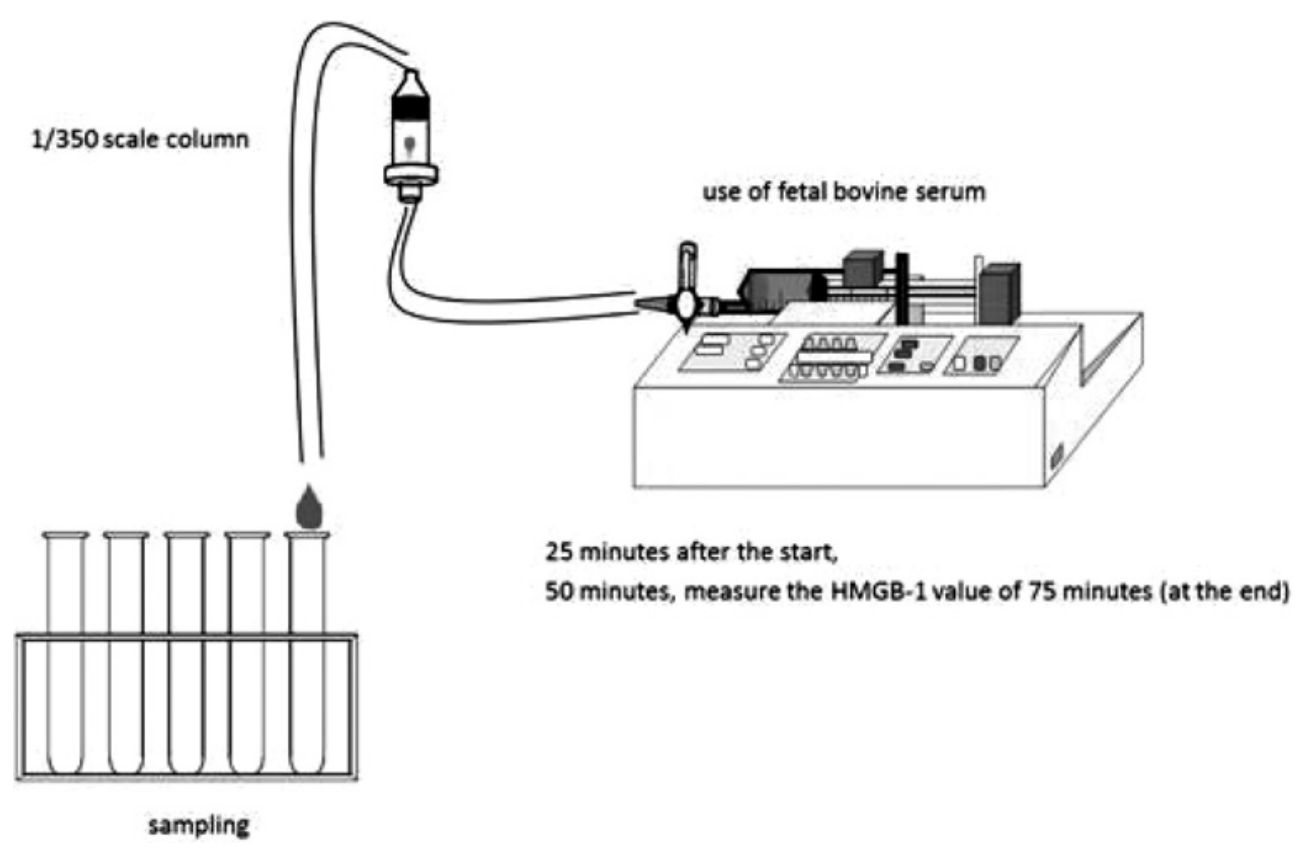

Fig. 2 A schematic representation of the experimental setup. We made 1/350 scales of clinically available columns for this study. We passed fetal bovine serum (FBS) through the column after priming with saline. The High Mobility Group Box-1 (HMGB-1) concentration in FBS of each column was examined at 25 minutes, 50 minutes, and 75 minutes following injection.

Table 1 The concentrations of plasma HMGB-1 (ng/mL) in each column

\begin{tabular}{lcccc}
\hline & 0 minutes & 25 minutes & 50 minutes & 75 minutes \\
\hline BRS & $63.0 \pm 11.0$ & $33.5 \pm 5.4$ & $34.9 \pm 5.5$ & $34.6 \pm 4.0$ \\
IM-PH & $62.8 \pm 10.0$ & $54.5 \pm 3.8$ & $56.4 \pm 4.7$ & $57.0 \pm 4.6$ \\
IM-TR & $61.3 \pm 9.2$ & $9.4 \pm 4.1$ & $18.3 \pm 5.6$ & $30.0 \pm 9.3$ \\
\hline
\end{tabular}

through a 1/350 scale column without plasma separation

(Fig. 2). HMGB-1 values of each sample were obtained by HMGB1 ELISA KitII (Shino-Test Corporation, Tokyo, Japan).

\section{Statistical Analysis}

All results are expressed as the mean values \pm standard deviations (SD). Differences in each value were analyzed by ONE-way ANOVA. All statistical analyses ware conducted using EZR (Saitama Medical Center of Jichi Medical University, Saitama, Japan) ${ }^{22}$. A p-value less than 0.05 was interpreted to be statistically significant.

\section{Results}

The highest adsorption of HMGB-1 was found in IM-TR, followed by BRS and IM-PH.

The actual concentrations of plasma HMGB-1 in each column are shown in Table $\mathbf{1}$ and the removal rate of HMGB-1 in each column was calculated with these values.
The removal rate of HMGB-1 in IM-TR was $86.3 \%$ (range, 77.8-92.4\%) at 25 minutes, 72\% (range, 63.9$79.9 \%$ ) at 50 minutes, and $54.2 \%$ (range, 39.5-65.3\%) at 75 minutes. The removal rates of HMGB-1 in BRS at each time point were $46.1 \%$ (range, 44.1-48.3\%), 43.8\% (range, $40.9-46.4 \%$ ), and $43.6 \%$ (range, 40.7-48.3\%), respectively. Finally, the removal rates of HMGB-1 in IM-PH at each time point were $9.6 \%$ (range, $-2.5-25 \%$ ), 6.6\% (range, $-9.2-20.8 \%$ ), and $5.5 \%$ (range, $-8.8-20 \%$ ), respectively

\section{(Fig. 3).}

In the IM-TR, the adsorption efficiency of HMGB-1 was approximately 90\% initially, but gradually declined toward the end of the experiment. Although BRS showed relatively lower adsorption efficiency than that of IM-TR, it maintained a steady removal rate of HMGB-1 at $45 \%$ throughout the experiment. IM-PH had the lowest adsorption efficiency, which was less than $10 \%$.

The total average removal rates at 75 minutes were $70.8 \%$ in the IM-TR, $44.5 \%$ in BRS, and $7.2 \%$ in the IM- 


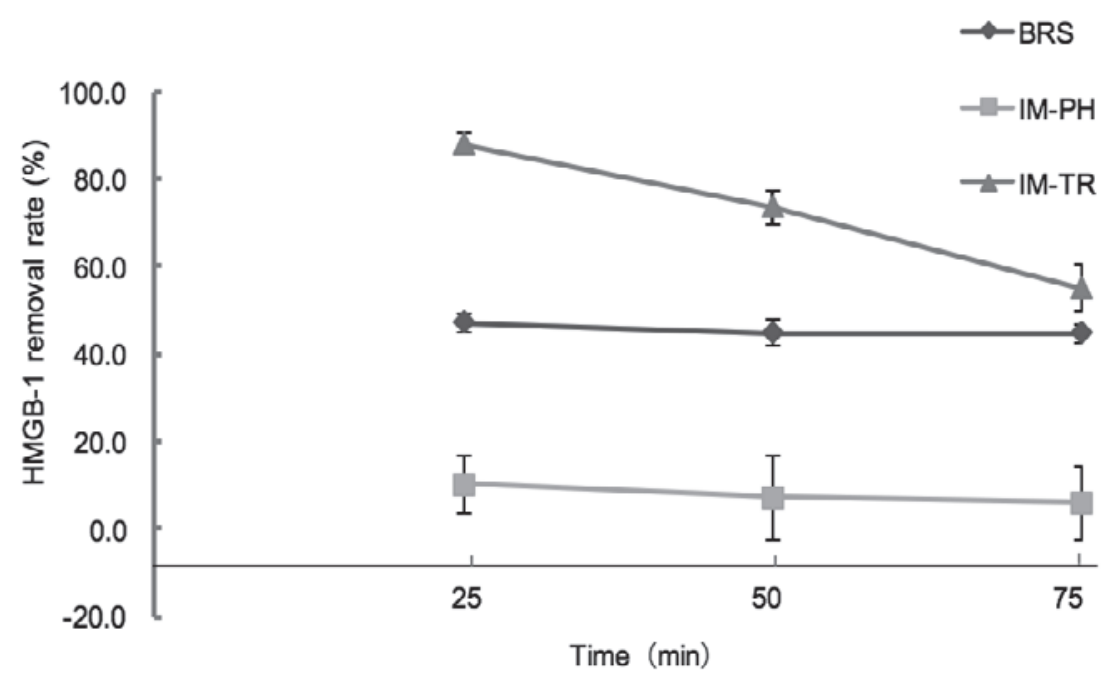

Fig. 3 The High Mobility Group Box-1 (HMGB-1) removal rates of each column are shown at 25 minutes, 50 minutes, and 75 minutes after the injection. The HMGB-1 removal rate of TR-350 (IM-TR) was about 90\% initially, but gradually declined. BRS-350 (BRS) maintained a removal rate of about $45 \%$ throughout the experiment. There was no difference in the HMGB-1 removal rate between IM-TR and BRS at 75 minutes, but all other cases showed significant differences.

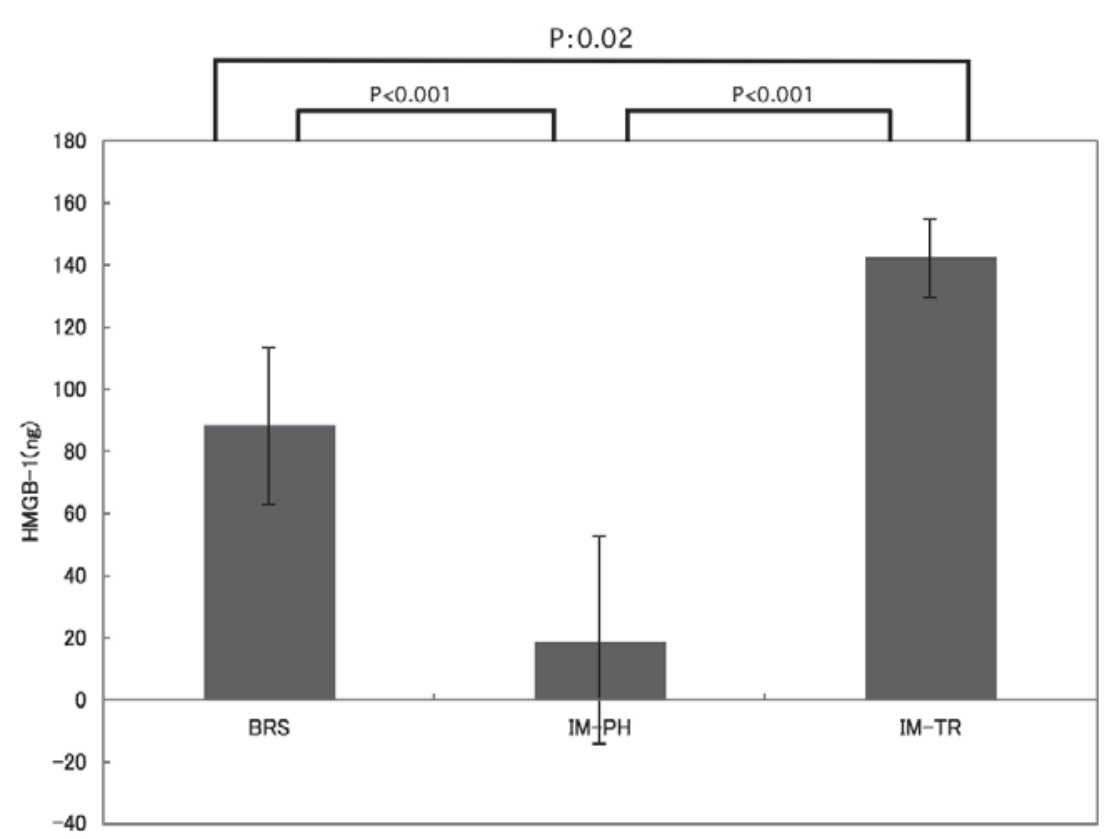

Fig. 4 The total High Mobility Group Box-1 (HMGB-1) removal amounts of each column are shown. The highest adsorption of HMGB-1 was observed in (TR-350) IM-TR, followed by BRS-350 (BRS) and (PH-350) IM-PH with statistical significance.

$\mathrm{PH}$ respectively. The average value of these three groups showed statistically significant differences at 25 minutes and 50 minutes in HMGB-1 removal capacity. There was no significant difference between IM-TR and BRS $(p=$ $0.54)$ at 75 minutes.

IM-TR removed the highest amount of total HMGB-1, followed by BRS and IM-PH, which removed the least. The removal amount of HMGB-1 in each column on average was $142.1 \mathrm{ng} / \mathrm{mL}$ for IM-TR, $88.2 \mathrm{ng} / \mathrm{mL}$ for BRS, and $20 \mathrm{ng} / \mathrm{mL}$ for IM-PH, which was statistically significant (Fig. 4). 


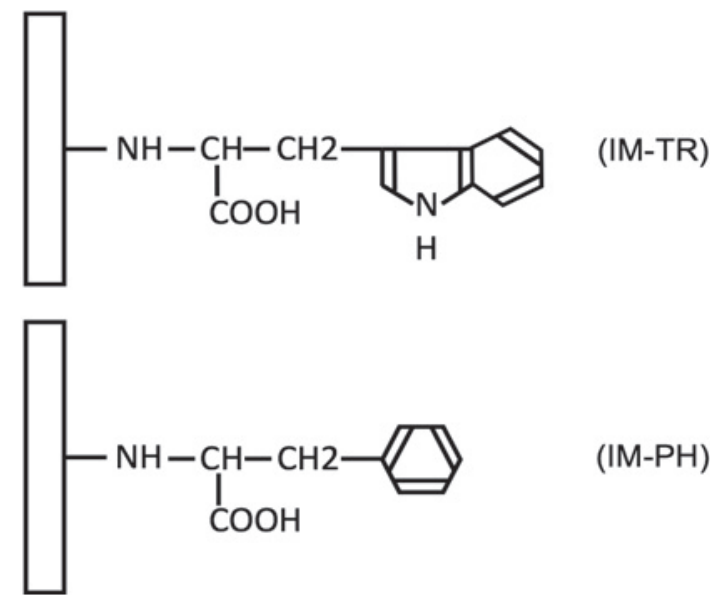

Fig. 5 The adsorption ligands of (TR-350) IM-TR and (PH-350) IM-PH are tryptophan and phenylalanine, respectively. The difference between tryptophan and phenylalanine in their acidity influenced the removal rate of High Mobility Group Box-1 (HMGB-1).

\section{Discussion}

In this study, we showed that HMGB-1 is adsorbed by the column which is used in plasma adsorption therapy.

HMGB-1 is a late and lethal mediator in severe sepsis and it is known to be secreted in two different pathways $^{8}$. It is passively secreted from necrotic cells and actively secreted from activated macrophages and platelets. The alarmin activates NF-kB via toll-like receptor (TLR)-2 and TLR4, resulting in amplification of inflammatory responses $^{3,23,24}$. TLRs on monocytes and macrophages are responsible for chain reaction events after infection. Once an infection is recognized, an inflammatory reaction cascade is activated ${ }^{25}$. First, endogenous cannabinoids which are immediate mediators of inflammation, such as anandamide (ANA) and 2-arachidonylglycerol (2-AG), are produced $^{26}$. These cannabinoids affect circulatory dynamics and regulate cytokine production ${ }^{27}$. It was reported that the endogenous cannabinoid was removed and adsorbed by PMX-DHP ${ }^{28,29}$. It is said that endogenous cannabinoids involved in the regulation of cytokine production are directly removed by PMX-DHP leading to an improvement of hemodynamics ${ }^{27,29}$, but the cytokine cannot be removed by PMX-DHP directly ${ }^{30}$.

HMGB-1 concentration in patients with septic shock strongly correlates with severity of the disease ${ }^{8}$. Although PMX-DHP and CHDF are well-developed blood purification therapies for sepsis in Japan, they are currently controversial as a treatment option. In DIC and multiple organ failure, HMGB-1 levels are significantly increased, which accelerates the chain reactions that lead to cy- tokine storm. Since PMX-DHP therapy does not directly reduce the amount of HMGB-1, its therapeutic effect on these severe conditions is limited ${ }^{31}$. However, PMX-DHP does adsorb early mediators in sepsis that are upstream of HMGB-1, which eventually decreases the concentration of HMGB-1. Also, many previous studies have reported that high anti-HMGB-1 antibody levels, which inhibit HMGB-1 activity, contribute to an improved survival rate r2-34. $^{32}$.

The IM-TR and IM-PH columns have a carboxyl group which is negatively charged and a hydrophobic group ${ }^{20,21}$. HMGB-1, which has two positively charged DNA binding regions, is one of the alarmins which has recently been reported to be related to various diseases ${ }^{35-37}$. HMGB-1 also has a hydrophobic protein, lysine, in its structure. The positively charged area of HMGB-1 binds to the negatively charged carboxyl group of the adsorption ligand by an ionic interaction in IM-TR and IM-PH. HMGB-1 was adsorbed in the adsorption ligand through a hydrophobic interaction in all three columns. Therefore, we presume that hydrophobic interaction might further enhance adsorption efficiency in IM-TR and BRS.

There was a difference in the adsorption capability between IM-TR and IM-PH. In this study, IM-TR was able to adsorb HMGB-1 significantly more than IM-PH. Based on the principles stated earlier, we hypothesize that it is due to the difference in the degree of hydrophobicity and acidity of the protein of the adsorption ligand. IM-TR is fixed with tryptophan, while IM-PH is fixed with phenylalanine $\mathrm{e}^{20}$. The adsorption capability of adsorbent is determined by a hydrophobic bond and an ionic bond. The larger the hydrophobicity of the hydrophobic amino acid of the ligand, the higher the adsorption rate of the substance. Tryptophan is more hydrophobic than phenylalanine $^{38}$. Therefore, IM-TR, which is fixed with tryptophan, theoretically has a stronger hydrophobic interaction with HMGB-1. Moreover, tryptophan, which is used in IM-TR, has an indole ring possessing a higher electrophilic aromatic effect than a benzene ring. Therefore, tryptophan is a strongly acidic amino acid due to the hydroxylation of its carboxyl group (Fig. 5).

BRS contains polystyrene as a main material, which is hydrophobic due to the phenyl group. HMGB-1 has a negatively charged region in its C-terminal. Therefore, we hypothesized that HMGB-1 was adsorbed in BRS through both ionic and hydrophobic interactions.

A previously published report showed that it is more reasonable to use charged membranes with adsorption capabilities in order to adsorb HMGB- $1^{15}$. Interestingly 
our data revealed that hydrophobicity is a more efficient interaction to adsorb HMGB-1 than charge in our columns. We successfully demonstrated that plasma adsorption membranes, especially IM-TR and BRS, were able to adsorb HMGB-1 efficiently for the first time. The limitations of our study include the fact that it was an in vitro experiment lacking in vivo confirmation and controls (e.g. comparison with PMX-DHP). However, since these plasma adsorption membranes have already been applied for clinical use, especially for autoimmune diseases, they should be equally safe and efficient for the treatment of severe sepsis.

\section{Conclusions}

To the best of our knowledge, this is the first study demonstrating HMGB-1 is captured by plasma adsorption.

Control of aberrant HMGB-1 levels by plasma adsorption therapy was successfully observed in the present study, and it can be applied to the treatment of sepsis as a new therapeutic option.

Acknowledgement: I would like to thank Dr. Janet Markman for her continued support in English editing.

Conflict of Interest: No conflict of interest declared.

\section{References}

1. Rittirsch D, Flierl MA, Ward PA: Harmful molecular mechanisms in sepsis. Nature Reviews Immunology 2008; 8: 776-787.

2. Takeuchi O, Akira S: Pattern recognition receptors and inflammation. Cell 2010; 140: 805-820.

3. Wang H, Yang H, Tracey K: Extracellular role of HMGB1 in inflammation and sepsis. Journal of internal medicine 2004; 255: 320-331.

4. Harris HE, Raucci A: Alarmin (g) news about danger. EMBO reports 2006; 7: 774-778.

5. Piccinini A, Midwood K: DAMPening inflammation by modulating TLR signalling. Mediators of inflammation 2010; 2010: 1-21.

6. Hatada $\mathrm{T}$, Wada $\mathrm{H}$, Nobori $\mathrm{T}$, Okabayashi $\mathrm{K}$, Maruyama K, Abe Y, Uemoto S, Yamada S, Maruyama I: Plasma concentrations and importance of High Mobility Group Box protein in the prognosis of organ failure in patients with disseminated intravascular coagulation. Thrombosis and haemostasis 2005; 94: 975-979.

7. Wang H, Zhu S, Zhou R, Li W, Sama AE: Therapeutic potential of HMGB1-targeting agents in sepsis. Expert Reviews in Molecular Medicine 2008; 10: 1-20.

8. Wang H, Bloom O, Zhang M, Vishnubhakat JM, Ombrellino M, Che J, Frazier A, Yang H, Ivanova S, Borovikova L: HMG-1 as a late mediator of endotoxin lethality in mice. Science 1999; 285: 248-251.

9. Bianchi ME: HMGB1 loves company. Journal of leukocyte biology 2009; 86: 573-576.

10. Marshall JC: Such stuff as dreams are made on: mediator- directed therapy in sepsis. Nature Reviews Drug Discovery 2003; 2: 391-405.

11. Tidswell M, Tillis W, LaRosa SP, Lynn M, Wittek AE, Kao R, Wheeler J, Gogate J, Opal SM, Group ESS: Phase 2 trial of eritoran tetrasodium (E5564), a toll-like receptor 4 antagonist, in patients with severe sepsis. Critical care medicine 2010; 38: 72-83.

12. Hirasawa $\mathrm{H}$ : Indications for blood purification in critical care. Acute Blood Purification 2010; 166: 21-30.

13. Dellinger RP, Levy MM, Rhodes A, Annane D, Gerlach H, Opal SM, Sevransky JE, Sprung CL, Douglas IS, Jaeschke R: Surviving Sepsis Campaign: international guidelines for management of severe sepsis and septic shock, 2012. Intensive care medicine 2013; 39: 165-228.

14. Oda S, Aibiki M, Ikeda T, Imaizumi H, Endo S, Ochiai R, Kotani J, Shime N, Nishida O, Noguchi T: The Japanese guidelines for the management of sepsis. Journal of intensive care 2014; 2: 55.

15. Yumoto M, Nishida O, Moriyama K, Shimomura Y, Nakamura T, Kuriyama N, Hara Y, Yamada S: In vitro evaluation of high mobility group box 1 protein removal with various membranes for continuous hemofiltration. Ther Apher Dial 2011; 15: 385-393.

16. Shibuya N, Sato T, Osame M, Takegami T, Doi S, Kawanami S: Immunoadsorption therapy for myasthenia gravis. Journal of Neurology, Neurosurgery \& Psychiatry 1994; 57: 578-581.

17. Seta T, Nagayama $H$, Katsura $K$, Hamamoto M, Araki T, Yokochi M, Utsumi K, Katayama Y: Factors influencing outcome in Guillain-Barré Syndrome: comparison of plasma adsorption against other treatments. Clinical neurology and neurosurgery 2005; 107: 491-496.

18. Noiri E: Choice of Apheresis Therapy. The Concise Manual of Apheresis Therapy, Springer. 2014; pp131-140,

19. Yoshida M, Tamura Y, Yamada Y, Yamawaki N, Yamashita $\mathrm{Y}$ : Immusorba TR and Immusorba PH: basics of design and features of functions. Therapeutic Apheresis and Dialysis 2000; 4: 127-134.

20. Hirata $N$, Kuriyama T, Yamawaki N: Immusorba $\mathrm{Tr}$ and Ph. Therapeutic Apheresis and Dialysis 2003; 7: 85-90.

21. Hirano R, Hirata N: Immunoadsorption using Immusorba TR and PH. Transfusion and apheresis science: official journal of the World Apheresis Association: official journal of the European Society for Haemapheresis 2017; 56: 661-665.

22. Kanda Y: Investigation of the freely available easy-to-use software 'EZR' for medical statistics. Bone marrow transplantation 2013; 48: 452-458.

23. Park JS, Gamboni-Robertson F, He Q, Svetkauskaite D, Kim J-Y, Strassheim D, Sohn J-W, Yamada S, Maruyama I, Banerjee A: High mobility group box 1 protein interacts with multiple Toll-like receptors. American Journal of Physiology-Cell Physiology 2006; 290: C917-C924.

24. Bianchi ME: DAMPs, PAMPs and alarmins: all we need to know about danger. Journal of leukocyte biology 2007; 81: 1-5.

25. Takeuchi O, Hoshino K, Kawai T, Sanjo H, Takada $\mathrm{H}$, Ogawa T, Takeda K, Akira S: Differential roles of TLR2 and TLR4 in recognition of gram-negative and grampositive bacterial cell wall components. Immunity 1999; 11: 443-451.

26. Varga K, Wagner JA, Bridgen DT, Kunos G: Platelet-and macrophage-derived endogenous cannabinoids are involved in endotoxin-induced hypotension. The FASEB Journal 1998; 12: 1035-1044.

27. Klein TW, Lane B, Newton CA, Friedman H: The can- 
nabinoid system and cytokine network. Proceedings of the Society for Experimental Biology and Medicine 2000; 225: 1-8.

28. Wang $Y$, Liu $Y$, Sarker KP, Nakashima M, Serizawa T, Kishida A, Akashi M, Nakata M, Kitajima I, Maruyama I: Polymyxin B binds to anandamide and inhibits its cytotoxic effect. FEBS letters 2000; 470: 151-155.

29. Kohro S, Imaizumi H, Yamakage M, Masuda Y, Namiki A, Asai Y, Maruyama I: Anandamide absorption by direct hemoperfusion with polymixin B-immobilized fiber improves the prognosis and organ failure assessment score in patients with sepsis. Journal of anesthesia 2006; 20: 1116.

30. Tani T: Review of endotoxin-adsorbing direct hemoperfusion therapy using a column containing polymyxin B immobilized fiber. Current Opinion in Critical Care 2000; 6: 416-420.

31. Yamato M, Minematsu $Y$, Fujii J, Mori K, Minato T, Miyagawa S, Fujimura R, Morikage N, Arata Y, Nakano C: Effective Combination Therapy of Polymyxin-B Direct Hemoperfusion and Recombinant Thrombomodulin for Septic Shock Accompanied by Disseminated Intravascular Coagulation: A Historical Controlled Trial. Therapeutic Apheresis and Dialysis 2013; 17: 472-476.

32. Yang H, Ochani M, Li J, Qiang X, Tanovic M, Harris HE, Susarla SM, Ulloa L, Wang H, DiRaimo R: Reversing established sepsis with antagonists of endogenous highmobility group box 1 . Proceedings of the National Academy of Sciences 2004; 101: 296-301.

33. Barnay-Verdier S, Fattoum L, Borde C, Kaveri S, Gibot S,
Maréchal V: Emergence of autoantibodies to HMGB1 is associated with survival in patients with septic shock. Intensive care medicine 2011; 37: 957-962.

34. Suda K, Kitagawa Y, Ozawa S, Saikawa Y, Ueda M, Ebina M, Yamada S, Hashimoto S, Fukata S, Abraham E: Antihigh-mobility group box chromosomal protein 1 antibodies improve survival of rats with sepsis. World journal of surgery 2006; 30: 1755-1762.

35. Merenmies J, Pihlaskari R, Laitinen J, Wartiovaara J, Rauvala $\mathrm{H}$ : $30-\mathrm{kDa}$ heparin-binding protein of brain (amphoterin) involved in neurite outgrowth. Amino acid sequence and localization in the filopodia of the advancing plasma membrane. Journal of Biological Chemistry 1991; 266: 16722-16729.

36. Lotze MT, Tracey KJ: High-mobility group box 1 protein (HMGB1): nuclear weapon in the immune arsenal. Nature Reviews Immunology 2005; 5: 331-342.

37. Cardin AD, Weintraub H: Molecular modeling of proteinglycosaminoglycan interactions. Arteriosclerosis, Thrombosis, and Vascular Biology 1989; 9: 21-32.

38. Fauchere J-L, Pliska V: Hydrophobic parameters pi of amino-acid side chains from the partitioning of N-acetylamino-acid amides. Eur J Med Chem 1983; 18: 369-375.

(Received, December 13, 2017)

(Accepted, January 12, 2018) 